\title{
Online Parameter Estimation for Surgical Needle Steering Model
}

\author{
Kai Guo Yan ${ }^{1}$, Tarun Podder $^{3}$, Di Xiao ${ }^{1}$, Yan $\mathrm{Yu}^{3}$, Tien-I Liu ${ }^{4}$, \\ Keck Voon Ling ${ }^{2}$, and Wan Sing $\mathrm{Ng}^{1}$ \\ Schools of ${ }^{1}$ MAE, ${ }^{2}$ EEE, Nanyang Technological University, Singapore \\ ${ }^{3}$ Department of Radiation Oncology, University of Rochester, NY, U.S.A. \\ ${ }^{4}$ Computer Integrated Manufacturing Lab, California State University, Sacramento, \\ California, U.S.A.
}

\begin{abstract}
Estimation of the system parameters, given noisy input/output data, is a major field in control and signal processing. Many different estimation methods have been proposed in recent years. Among various methods, Extended Kalman Filtering (EKF) is very useful for estimating the parameters of a nonlinear and time-varying system. Moreover, it can remove the effects of noises to achieve significantly improved results. Our task here is to estimate the coefficients in a spring-beam-damper needle steering model. This kind of spring-damper model has been adopted by many researchers in studying the tissue deformation. One difficulty in using such model is to estimate the spring and damper coefficients. Here, we proposed an online parameter estimator using EKF to solve this problem. The detailed design is presented in this paper. Computer simulations and physical experiments have revealed that the simulator can estimate the parameters accurately with fast convergent speed and improve the model efficacy.
\end{abstract}

Keywords: Online parameter estimation, spring/damper model, EKF.

\section{Background}

Precise needle insertion is very important for a number of percutaneous interventions. Yet it's very difficult to achieve in real practice e due to tissue heterogeneity and elastic stiffness, unfavorable anatomic structures, needle bending, inadequate sensing, tissue/organ deformation and movement, and poor maneuverability. Several research groups (e.g. [1,2]) have implemented computer and robotic assistance to align the needle with the target assuming known target location in $3 \mathrm{D}$, target immobilization, and a straight needle path. Errors caused by needle deflection and tissue deformation have been observed for a long time. Yet to date, there are few effective physicalbased needle steering systems exist for correcting the needle deflection when it's observed. In addition, many procedures are currently not amenable to needles because of obstacles, such as bone or sensitive tissues, which lie between feasible entry points and potential targets. Thus, there is a clear motivation for needle steering in order to provide accurate and dexterous targeting. 
To solve this problem, a spring-beam-damper needle steering model has been developed [3], in which the tissue is represented by a series of spring-damper system. The proposed spring-beam-damper model takes into consideration both the linear and nonlinear tissue reaction effects when interacting with the flexible needle. In the literature, the spring-damper model has been adopted by many research groups [4-7] in studying the tissue deformation. One difficulty in using such model is to estimate the spring and damper coefficients of the tissue, especially when applying to a human in a real medical surgery.

This paper aims to design an effective parameter estimator for the spring-damper model we have developed. Considering the steering model is nonlinear, time varying, and at the same time, the dataset collected is very noisy, here we proposed an online parameter estimator using EKF to estimate the coefficients in real time. The detailed design is presented in this paper. Extensive computer simulations have been carried out to assess the efficacy of the proposed estimator. Results and conclusions are presented in the following sections.

\section{Design of the Online Parameter Estimator}

\subsection{The Dynamic Equations of Needle Steering Model}

This section briefly reviews the spring-beam-damper model we have proposed (refer to [3] for more details). Fig. 1 shows the mechanism of the needle steering procedure. The flexible needle is assumed to follow the Bernoulli-Euler beam model and to be clamped tightly at end. The tissue is represented by a series of spring-damper system. The initial lengths of springs are decided by the needle tip trajectory. At the beginning, the needle is placed next to the tissue. As the needle inserts into the tissue, the springs and dampers come into contact with the needles body and exert force on it. The forces of the springs at time instant $t$ are determined by the needle body shape at that time and the forces of the dampers are determined by the velocities of the contact points.

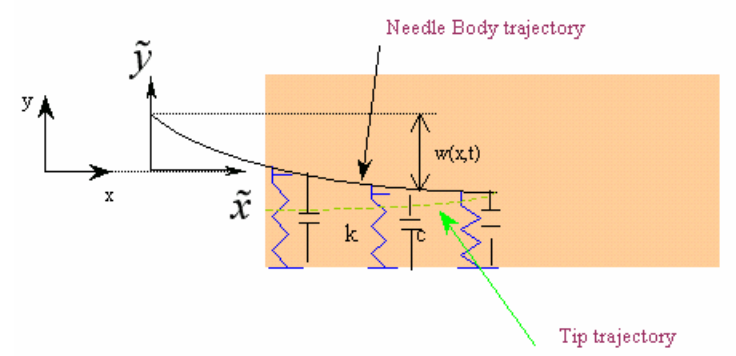

Fig. 1. Mechanism of the needle steering procedure 
The system dynamic equation can be derived using Hamilton's principle as follows:

$$
\int_{t_{1}}^{t_{2}}\left(\delta T-\delta V+\delta W_{n c}\right) d t=0
$$

with $T, V, W_{n c}$ representing the kinetic energy, potential energy and work done by non-conservative forces respectively.

Unconstrained modal analysis is adopted in solving the partial differential equations. By unconstrained modal analysis [8], the deflection of the beam at $\tilde{x}$, $\omega(\tilde{x}, t)$ and the position of the fixture, $y(t)$ can be represented, respectively, as

$$
\begin{gathered}
y=\alpha(t)+\beta q(t) \\
\omega(\tilde{x}, t)=\phi(\tilde{x}) q(t)
\end{gathered}
$$

where $\alpha(\mathrm{t})$ describes the motion of the center of mass of the total system without perturbation, $\phi(\tilde{x})$ is the shape function, $q(t)$ is the time-varying amplitude of motion. $\beta$ is defined to satisfy

$$
\beta=-\frac{\rho}{M_{t}} \int_{0}^{L} \phi(\tilde{x}) d \tilde{x}
$$

This allows transforming the nonhomogeneous equations into a set of second-order ordinary differential equations. After some algebraic manipulations, the model is finally derived as,

$$
\begin{aligned}
& \dot{\alpha}(t) \int_{L-v_{x} t}^{L} c \varphi_{i}(\tilde{x}) d \tilde{x}+\alpha(t) \int_{L-v_{x} t}^{L} k_{0} \varphi_{i}(\tilde{x}) d \tilde{x}+\ddot{q}_{i}(t)+\sum_{j} \dot{q}_{j}(t) \int_{L-v_{x} t}^{L} c \varphi_{i}(\tilde{x}) \varphi_{j}(\tilde{x}) d \tilde{x} \\
& +\sum_{j} q_{j}(t) \int_{L-v_{x} t}^{L} k_{0} \varphi_{i}(\tilde{x}) \varphi_{j}(\tilde{x}) d \tilde{x}+\omega_{i}^{2} q_{i}(t)=F_{y} \beta_{i}+f_{1 i} \\
& M_{t} \ddot{\alpha}(t)+c v_{x} \dot{\alpha}(t) t+\sum_{j} \dot{q}_{j}(t) \int_{L-v_{x} t}^{L} c \varphi_{j}(\tilde{x}) d \tilde{x}+\sum_{j} q_{j}(t) \int_{L-v_{x} t}^{L} k_{0} \varphi_{j}(\tilde{x}) d \tilde{x}+k_{0} v_{x} \alpha(t) t \\
& =F_{y}+f_{2}
\end{aligned}
$$

where,

$$
\begin{aligned}
M_{t} & =M+\rho L \\
f_{1 i} & =\int_{0}^{t} k_{0} \alpha\left(t_{1}\right) \varphi_{i}\left(v_{x} t_{1}+L-v_{x} t\right) v_{x} d t_{1}+\int_{0}^{t} k_{0} \sum_{j} \varphi_{j}(L) q_{j}\left(t_{1}\right) \varphi_{i}\left(v_{x} t_{1}+L-v_{x} t\right) v_{x} d t_{1} \\
& =\int_{0}^{t} k_{0} Y\left(t_{1}\right) \varphi_{i}\left(v_{x} t_{1}+L-v_{x} t\right) v_{x} d t_{1} \\
f_{2} & =\int_{0}^{t} k_{0} \alpha\left(t_{1}\right) v_{x} d t_{1}+\int_{0}^{t} k_{0} \sum_{j} \varphi_{j}(L) q_{j}\left(t_{1}\right) v_{x} d t_{1}=\int_{0}^{t} k_{0} Y\left(t_{1}\right) v_{x} d t_{1}
\end{aligned}
$$


here, $\mathrm{M}$ is the mass of the fixture, which links the needle with the 3D motion platform. $\mathrm{M}_{\mathrm{t}}$ is the total mass of the fixture and needle. $\mathrm{Y}(\mathrm{t} 1)$ is the needle tip position at time instant $\mathrm{t} 1 . \mathrm{F}_{\mathrm{y}}$ is the steering force, which is acted at the needle end in $\mathrm{y}$ direction. $\mathrm{V}_{\mathrm{x}}$ is needle insertion speed. $\mathrm{L}$ is the length of the elastic beam. $i$ and $j$ here refer to different mode shape. We consider continuous distributed spring and dampers along the needle body. So here $\mathrm{k}_{0}$ is stiffness coefficient of the spring per unit length, and $\mathrm{c}$ is the damping coefficient per unit length. At current stage, $\mathrm{k}_{0}$ and $\mathrm{c}$ are assumed to be constant.

\subsection{Design of the Parameter Estimator}

Estimation of the system parameters, given noisy input/output data, is a major field in control and signal processing. Many different estimation methods have been proposed in recent years [9-13]. Among various methods, Extended Kalman filtering (EKF) is very useful for estimating the parameters of a nonlinear and time-varying system. And at the same time, it can remove the effects of interferences and noises to achieve satisfactory results. Considering the needle steering model is nonlinear and time varying, and furthermore, the data we obtain are usually very noisy, we adopt EKF method to design the parameter estimator.

The steering model is rearranged in the state-space form. Here the model spring and damper coefficients are treated as states too. For simplicity, we express the needle steering model as

$$
\dot{x}=g(x)+w
$$

$w$ is a random zero-mean process. The measurement equation, the output tip position is a linear function of the states. Because the measurements are discrete, we write the equation as

$$
y_{k}=c x_{k}+v
$$

where $c=\left[\begin{array}{llllll}1 & \phi(L) & 0 & 0 & 0 & 0\end{array}\right]$ and $v$ is a zero-mean random process.

Because the system equations are nonlinear, a first-order approximation is used in the continuous Riccati equations for the systems dynamics matrix $F$. The matrices are related to the nonlinear system according to

$$
F=\left.\frac{\partial g(x)}{\partial x}\right|_{x=\tilde{x}}
$$

The fundamental matrix, required for the discrete Riccati equations, can be approximated by the Taylor-series expansion. In this application, the fundamental matrix will only be used in the calculation of the Kalman gains. As demonstrated in [14] that adding more terms to the Taylor-series expansion for the fundamental matrix may not improve the performance of the overall filter, here only the first two terms are used to approximate the series. 


$$
\Phi_{k} \approx I+F T_{s}
$$

The Riccati equations required for the computation of the Kalman gains, are [14]

$$
\begin{aligned}
& M_{k}=\Phi_{k} P_{k-1} \Phi_{k}^{T}+Q_{k} \\
& K_{k}=M_{k} C^{T}\left(C M_{k} C^{T}+R\right)^{-1} \\
& P_{k}=\left(I-K_{k} C\right) M_{k}
\end{aligned}
$$

here, $\Phi_{k}$ is the fundamental matrix, $P_{k}$ is a covariance matrix representing errors in the state estimates after an update and $M_{k}$ is the covariance matrix representing errors in the state estimates before an update. C is the coefficient matrix of output. Because $\Phi_{k}$ is nonlinear function of the state estimates, the Kalman gains cannot be computed offline as is possible with the linear kalman filter. $Q_{k}$ and $R$ are the process-noise matrix and measurement noise matrix, respectively. The discrete process-noise matrix $Q_{k}$ can be found from the continuous process-noise matrix according to

$$
Q_{k}=\int_{0}^{T_{s}} \Phi(\tau) Q \Phi^{T}(\tau) d t
$$

With an extended Kalman filter, the new state estimate is the old state estimate projected forward to the new sampling or measurement time plus a gain times a residual or

$$
\tilde{x}_{k}=\bar{x}_{k}+K_{k}\left[y_{k}-\bar{y}_{k}\right]
$$

here $K_{k}$ is the Kalman gain, $y_{k}$ is the measured output and $\bar{y}_{k}$ is the estimated output. The old estimates that have to be propagated forward do not have to be done with the fundamental matrix but instead can be propagated directly by integrating the actual nonlinear differential equations forward at each sampling interval.

\section{Validation of the Parameter Estimator}

This parameter estimator has been implemented using Matlab 7.0. To validate whether this estimator can estimate the parameter fast and accurately, firstly we used the pre-programmed model generator to generate the output needle tip position data with input force $10^{-4}(\mathrm{~N})$, the spring and damper coefficients per length $2\left(\mathrm{~N} / \mathrm{m}^{2}\right)$ and $5\left(\mathrm{~N} . \mathrm{s} / \mathrm{m}^{2}\right)$, respectively. Then these input and output data were filled into the parameter estimator. The initial states are chosen to be $\left[10^{-7} ; 10^{-7} ; 10^{-7} ; 10^{-7} ; 1 ; 4\right]$. The initial covariance matrix is estimated to be $\operatorname{diag}\left(\left[\begin{array}{llllll}0 & 0 & 0 & 0 & 4 \mathrm{e}+5 & 5 \mathrm{e}+5\end{array}\right]\right)$. The simulation results have been presented in Fig. 2 and Fig. 3. The RMS error for output is 6.9888e006. We can see that the estimator can estimate the coefficients accurately with fast convergent speed. 
The initial covariance matrix is very important for the convergent rate. Reasonable chosen initial covariance can improve the convergent rate, as shown in Fig. 4. Generally speaking, the convergent rate is increased with larger covariance matrix. But if the covariance matrix is set to be too large, the estimation will fluctuate and overshoot, as shown in Fig. 4. The setting of the covariance matrix is rather rule-ofthumb. It has been found during experiments that, for the last two terms in the initial covariance matrix, the magnitudes of $10^{5}$ can always give good convergence rate. Exceeding this magnitude may result in fluctuation and overshoot.
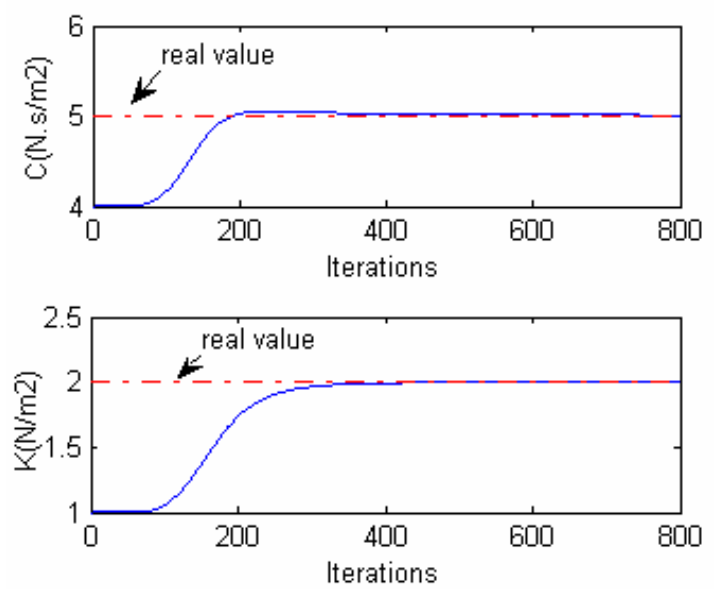

Fig. 2. Estimated damper \& spring coefficient vs. real value
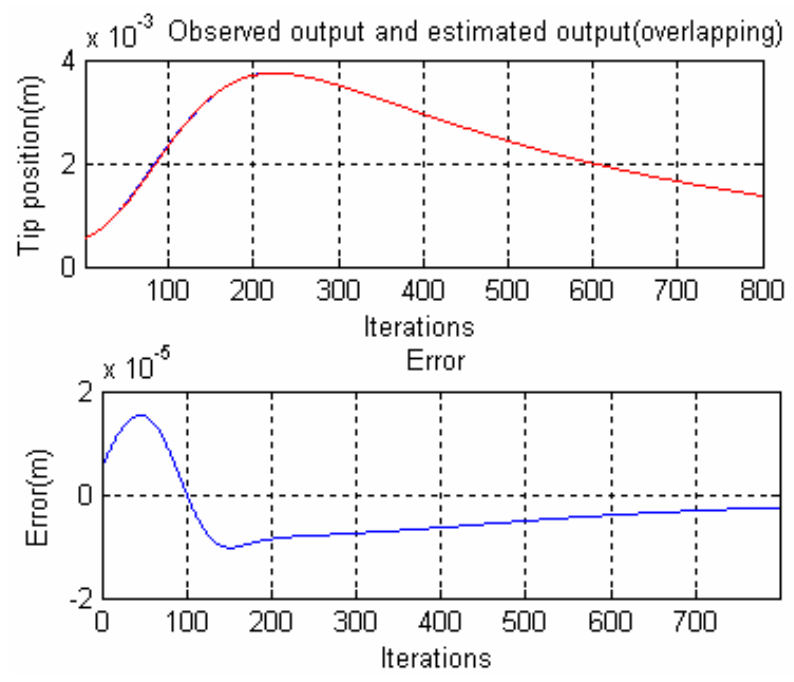

Fig. 3. Estimated needle tip position vs. measured tip position 

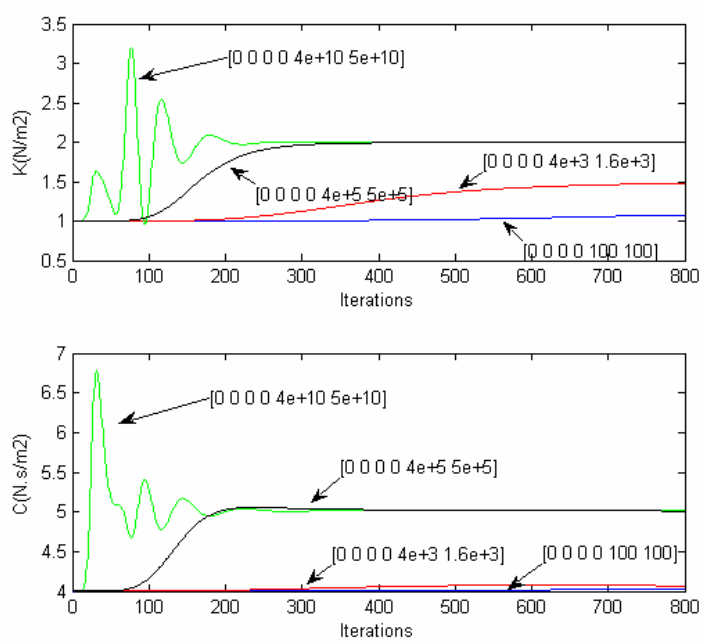

Fig. 4. Coefficient estimation comparison with different covariance matrixes

\section{Validation of the Steering Model Using Parameter Estimator}

The design purpose of this parameter estimator is to validate the parameters of the needle steering model. These parameters will be set into the steering model to do the simulation. The simulated output will then be compared with the measured output to validate the effectiveness of the model. For this purpose, physical experiment has been carried out. The experimental setup shown in Fig. 5 was used to carry out the experiment. The 3 Degree-of-Freedom (DOF) motion platform drives the needle into the

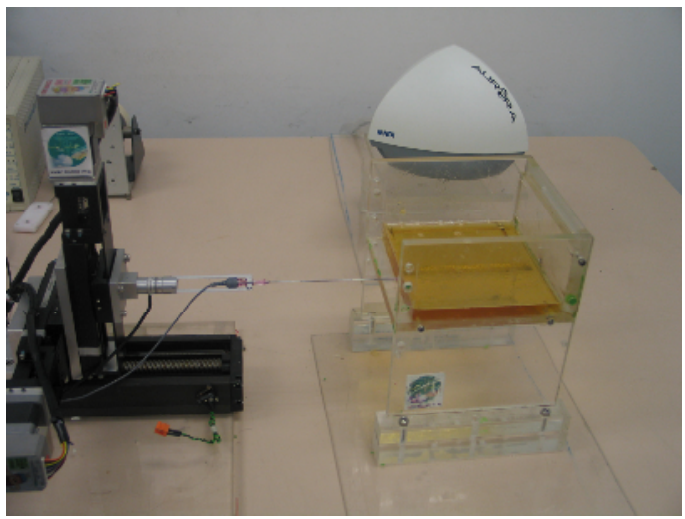

Fig. 5. Experiment setup phantom/animal organ following some pre-designed trajectory. A 6 DOF force/torque (F/T) sensor (Nano17-SI-12-0.12 ( from ATI Industrial Automation, USA) is mounted at the needle end to measure the needle end force. The needle adopted here is a 5-DOF MagTrax Needle Probe (from Traxtal Technology, Canada). It is a $130 \mathrm{~mm}$ long needle and has a sensor located at the stylet's proximal tip. This needle movement can be observed in real time via an electromagnetic system called Aurora ${ }^{\circledR}$ (from Northern Digital Inc. Canada).

Phantoms made of different gelatin/water ratios were firstly adopted to simulate the soft tissue, for it's easier to obtain and the properties are easier to control. The 
needle was driven into the phantoms with insertion speeds at $10 \mathrm{~mm} / \mathrm{s}, 8 \mathrm{~mm} / \mathrm{s}, 6 \mathrm{~mm} / \mathrm{s}$ and $4 \mathrm{~mm} / \mathrm{s}$, respectively. The needle tip trajectory and the end force were collected during the insertion. The input force data and output tip position data were filled into the estimator first to estimate the parameters. The estimated parameters and input force data were then put into the model for simulation. For the space constraint, only one result is shown here. The simulation result vs. the tracked output is shown in Fig. 6. The big drop-down at the beginning was caused by the sensor noise when the needle was accelerated to reach the set constant velocities. We can see that the proposed model has predicted the needle tip trajectory quite well.

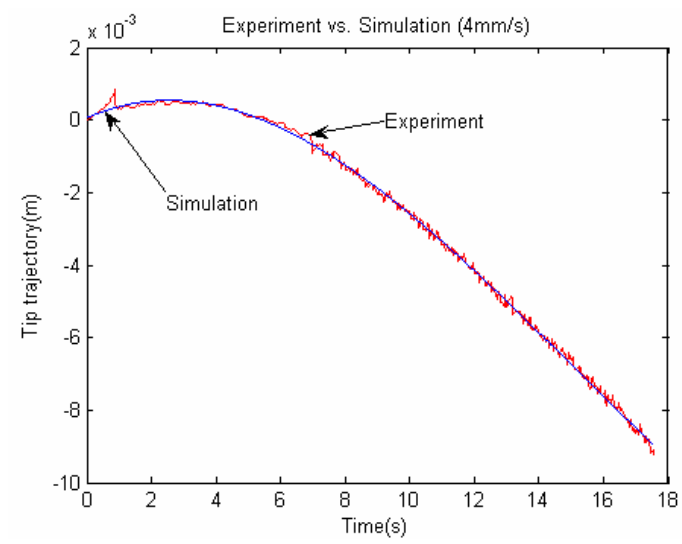

Fig. 6. Experiment vs. Simulation

\section{Conclusions and Future Works}

In this paper, we proposed an online parameter estimator using EKF for parameter estimation of the needle steering model. Simulation has been carried out first to validate this estimator before used for the steering model. Results have shown that the simulator can estimate the parameters fast and accurately with reasonably set initial covariance matrix. Even though the setting of the initial covariance matrix is rather rule-of-thumb, it has shown that, the magnitudes of $10^{5}$ can always give good convergence rate. Exceeding this magnitude may result in fluctuation and overshoot.

The steering model was then validated though physical experiment in gelatin phantom. The needle end force and needle tip position data were collected and put into the parameter estimator to estimate the parameters. The EKF method makes use of the minimum variance estimation that is optimal for each point in time, not a sum over time. This reversely ascertains that the output of the steering model when using the estimated parameters can generate the output that is the best fit for the measured output.

At current stage, the spring and damper coefficients are assumed to be constant. Considering the inhomogeneous human tissue, in the near future, we will improve them to be depth varying and modify the online parameter estimator accordingly for more robust performance. 
Acknowledgments. Nanyang Technological University (Singapore), NMRC 0859/2004 and US National Cancer Institute (NCI) (under grant R01 CA091763) are acknowledged for support.

\section{References}

1. Loser, M, Navab, N: A New Robotic System for Visually Controlled Percutaneous Interventions under CT. MICCAI. Lecture notes in computer science, Vol 1935, Springer Verlag, (2000)

2. Stoianovici, D., Cadeddu, J. A., Demaree, R. D., Basile, H. A., etc.: An Efficient Needle Injection Technique and Radiological Guidance Method for Percutaneous Procedures. Lecture Notes in Computer Science, Vol. 1205, Springer Verlag, (1997)

3. Kaiguo, Yan, Wan Sing Ng, Yan Yu, Tarun Podder, Tien-I Liu, CWS Cheng, Keck Voon Ling: Needle Steering Modeling and Analysis using Unconstrained Modal Analysis. BIOROB, Italy, (2006)

4. D. Terzopoulos and K. Waters: Analysis and Synthesis of Facial Image Sequences using Physical and Anatomical Models. IEEE Transactions on Pattern Analysis and Machine Intelligence, Vol. 15, (1993)

5. F. Boux de Casson and C. Laugier: Modeling the Dynamics of a Human Liver for a Minimally Invasive Surgery Simulator. MICCAI, Lecture Notes in Computer Science, Vol.1679, (1999)

6. Webster R: Elastically Deformable 3D Organs for Haptic Surgical Simulation. Proceedings of Medicine Meets Virtual Reality, Newport Beach, (2002)

7. P. F. Neumann, L. L. Sadler, and J. G. M.D: Virtual Reality Vitrectomy Simulator. MICCAI, Lecture Notes in Computer Science, Vol.1496, (1998)

8. Carlos Canudas de Wit, Bruno Siciliano and Georges Bastin: Theory of Robot Control, Springer, New York, (1996)

9. Martin Morf and Thomas Kailath: Square-Root Algorithms for Least-Squares Estimation. Transaction on Automatic Control, Vol. AC-20, (1975)

10. Mi Lu and Xiangzhen Qiao: Parallel Computation of the Modified Extended Kalman Filter. International Journal of Computer Math, Vol. 45, (1992)

11. Satoko Abiko, Kazuya Yoshida: On-line Parameter Identification of a Payload Handeled by Flexible Based Manimulator. Proceedings of 2004 IEEE/RSJ International Conference on Intelligent Robots and Systems, Japan, (2004)

12. Tien Li Chia, Po-Chuan Chow, Howard Jay Chizeck: Recursive Parameter Identification of Constrained Systems: An Application to Electrically Stimulated Muscle. IEEE Transactions on Biomedical Engineering, Vol. 38, (1991)

13. Akihiko Kumagai, Tien-I Liu, and Paul Holzian: Control of Shape Memory Alloy Actuators with A Neuro-Fuzzy Feedforward Model Element, Journal of Intelligent Manufacturing, Vol. 17, 45-56, (2006)

14. Paul Zarchan and Howard Musoff: Fundamentals of Kalman Filtering, A Practical Approach, the American Institute of Aeronautics and Astronautics, Inc.1801 Alexander Bell Drive, Reston, Virginia 20191-4344,Vol. 190, (2000) 